\title{
A Fast Real Time Link Adaptation Scheme for Wireless Communication Systems ${ }^{\star}$
}

\author{
Hyukjun $\mathrm{Oh}^{1}$, Jiman Hong ${ }^{2}$, and Yongseok Kim ${ }^{3, \star \star}$ \\ ${ }^{1}$ Kwangwoon University, Seoul, Korea \\ hj_oh@kw.ac.kr \\ ${ }^{2}$ Soongsil University, Seoul, Korea \\ jiman@ssu.ac.kr \\ ${ }^{3}$ Samsung Electronics, Suwon, Korea \\ yongseokkimasi@samsung.com
}

\begin{abstract}
In this paper, a fast real time link adaptation scheme for wireless communication systems is proposed. The proposed scheme is employing multi-stage adaptation controls based on channel state information (CSI). The optimal link adaptation scheme is known to have high complexity to implement, and it shoud be run iteratively to deal with time varying wireless channels in a short time. The proposed method determines the speed of channel variation using CSI's, then it applies the most appropriate link adaptation methodology or algorithm to the current time varying rate of channels. For examples, a simple up/down transmit power adjustment is used for the very fast time varying channel condition. On the other hand, the optimal link adaptation scheme is directly applied in the very slow varying channel state. The proposed multi-stage link adaptation scheme can be easily implemented in real time wirelss communication systems because of its capability of selecting an appropriate real time link adaptation scheme adaptively to each channel variation rate. The design example on the selected platform shows that the proposed scheme is very efficient in real time applications of the link adaptation in wireless communications, while its performance is maintained close to the optimal.
\end{abstract}

\section{Introduction}

Efficient utilization and allcation of the resources over the propagation channel is certainly one of the major challenges in wireless communication system design [1]. Especially, a modern day wireless communications system is required to operate over channels that experience fading and multipath. Given the evergrowing demand for wireless communication, a higher efficiency, higher performance wireless communications system is desirable. In order to improve the efficiency and decrease the complexity of the system, CSI's can be transmitted

\footnotetext{
* This research work has been supported by Seoul R\&BD Program in 2007.

** Corresponding author.
} 
back to the transmitter unit in order to precondition the signal before transmission. These preconditioning or transmit coordination works are called link adaptations.

One such communications system is an orthogonal frequency division multiplex (OFDM) system with CSI feedbacks. Its multicarrier nature with CSI feedbacks allows the use of link adaptation to enhance its performance significantly 1, 2. The idea of link adaptation in OFDM is as follows. Based on the channel characteristic given by CSI's, the number of bits to be transmitted, the level of modulation and the transmission power in each sub-carrier are selected in order to increase the transmission bit rate [3, 4] or reduce the required transmit power 5 .

For example, in a standard TDMA system employing OFDM with link adaptation, each user is allocated a fixed time slot and only that user can transmit in that time slot. These unused sub-carriers, as a result of link adaptation, are wasted and cannot be used by other users during that time slot. However, the sub-carriers that are in deep fade over the link between the base station (BS) and the designated mobile user may not be in deep fade over the links between the BS and other mobile users. This motivates us to consider the link adapation schemes that users share the downlink transmission by adaptively using different sub-carriers, instead of using different time slots as in a TDMA system. This approach will allow all the sub-carriers to be used effectively. However, its algorithm is not simple to implement like one proposed in [6] that is not suitable for the time varying channel as the required computation makes real-time implementation impractical.

The optimal link adaptation strategy is to perform link adaptation schemes like power allocation, sub-carrier allocation, bit allocation, user allocation, modulation and coding allocation every time the propagation channel condition is changed. Unfortunately, however, it is quite difficult to perform the optimal link adaptation strategy in the fast time varying channel condition, because its complexity is pretty high to be run in real time.

In this work, we propose a link adaptation scheme that is simple to implement. Link adaptation strategy is developed which results in minimum transmit power. Also real-time heuristic allocation algorithm which is close to the optimum while having much fewer computations is also proposed. The proposed scheme is employing multi-stage adaptation controls as per channel variation rate based on channel state information (CSI). The proposed method determines the speed of channel variation using CSI's, then it applies the most appropriate link adaptation methodology or algorithm to the current time varying rate of channels. For examples, a simple up/down transmit power adjustment is used for the very fast time varying channel condition. On the other hand, the optimal link adaptation scheme is directly applied in the very slow varying channel state. The proposed multi-stage link adaptation scheme can be easily implemented in real time wirelss communication systems because of its capability of selecting an appropriate real time link adaptation scheme adaptively to each channel variation rate. We compare the performance and complexity of these algorithms with existing ones to 
show the effectiveness and efficiency of the proposed scheme. Such design examples on the selected platform shows that the proposed scheme is very efficient in real time applications of the link adaptation in wireless communications, while its performance is maintained close to the optimal.

\section{System Model}

The structure of a multiuser OFDM system using link adaptation is shown in Fig. 1].

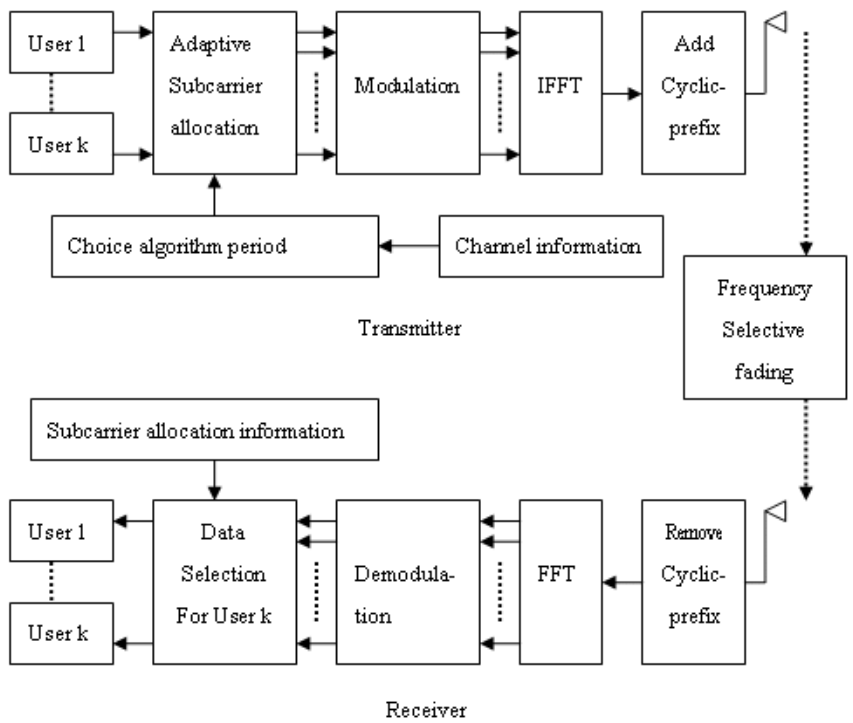

Fig. 1. System model of OFDM system with link adaptation

Let $K$ be the number of users supported by the system and $N$ be the number of sub-carriers. Also, let $S_{k}$ denote the number of sub-carriers required by user $k$. Assume each sub-carrier has a bandwidth that is much smaller than the coherence bandwidth of the channel and the instantaneous channel gain of each sub-carrier for every user is known to the base station. In the transmitter side, the link adaptation algorithm assigns sub-carriers to each user according to the channel gains. The bit stream of each user is then transmitted out using the assigned sub-carriers. In each sub-carrier, the assigned bits are modulated with a power level that can overcome the fading of the channel. In addition to the power required to transmit the bits in the AWGN channel, power proportional to the inverse of the corresponding sub-carrier channel gain $a_{k}$," is needed. The modulated signals of all the sub-carriers are then transformed into time domain using IFFT and cyclic prefix is inserted to eliminate the ISI. Dedicated sub-carriers are reserved for the broadcasting of the sub-carrier allocation or link adaptation 
information of which each receiver can retrieve to determine the corresponding sub-carriers for receiving the data. In the receiver side, after removing the guard interval and transforming the time domain samples into the frequency domain symbol, the user demodulates the sub-carriers prescribed by the link adaptation of allocation information to recover the original bit stream. The goal of the link adaptation is to minimize the total transmit power of all the users while satisfying the data transmission requirement for each user. Given $P$ is the transmit power of a sub-carrier in the AWGN channel and $p_{k, n}$ is a binary assignment variable which is equal to 1 if sub-carrier $n$ is assigned to user $k$ or equal to 0 otherwise, the total transmission power is given by

$$
P_{T}=\min \sum_{n=1}^{N} \sum_{k=1}^{K} p_{k, n} \frac{P}{\alpha_{k, n}^{2}}
$$

The objective is to find the values of the assignment variables, $p_{k, n}$, to minimize $p_{T}$ while satisfying the following constraints,

$$
\begin{aligned}
& \sum_{n=1}^{N} p_{k, n}=S_{k}, \quad k \in\{1,2, \ldots, K\} \\
& \sum_{n=1}^{N} p_{k, n}=1, \quad n \in\{1,2, \ldots, N\} .
\end{aligned}
$$

Constraint (2) specifies that the total number of sub-carriers allocated to user $k$ is $S_{k}$ and constraint (3) specifies that each sub-carrier can only be allocated to one user.

\section{Optimal Link Adaptation}

The above optimization problem is similar to the classical assignment problem in the area of linear programming. Therefore, we can solve this problem by first transforming it into an equivalent assignment problem. Here each user is expanded to $S_{k}$ sub-users and for every sub-user, only one sub-carrier will be allocated. The channel gain of the sub-user is equal to that of the original user. The optimization problem then becomes Eq. (1) with $K=N$. The constraints (2) are also transformed to $\sum_{n=1}^{N} p_{k, n}=1$ with $K=N$.

Numerous methods have been proposed to solve this classical assignment problem. One of them is the famous Hungarian method proposed in [7] of which the computation complexity of the algorithm is $O\left(n^{4}\right)$. In a time varying channel, the channel characteristic of users changes frequently. To cope with this situation, we need a link adaptation algorithm like a sub-carrier allocation above that is fast enough to allocate the subcarrier within the coherence time of the channel. Unless it is fast enough, the actual link adaptation operation cannot be run in real time while keeping achieving the required performance. The optimal 
link adaptation strategy is to run the optimal link adaptation algorithm like the sub-carrier allocation given in above continuously as per channel variations. Ideally, the link adaptation should be done before the channel varies. It is quite difficult goal to achieve and it is even impossible for fast varying channels with high speed mobiles. That is, the complexity of the above optimal algorithm is too large that an optimal solution may not be generated within the coherence time, especially for a fast-fading channel. Therefore, we propose a heuristic link adaptation scheme that satisfies the real time requirement.

\section{Proposed Multi-stage Link Adaptation Strategy}

The optimal link adaptation algorithm of sub-carrier and transmit power allocations addressed in the previous section is not appropriate for the real time operation due to its high complexity and duty cycle limitation in fast varying channel condition. In this section, we propose using CSI's to determine the current channel variation rate and propose to use the computationally efficient link adaptation alogorithm optimized for the estimated speed of channel variations.

The channel variation rate is not required to be very accurate because the characteristics of the propogation channel is not changing rapidly as per the channel variation rate. Its characteristics are more dependent on the trend of channel variation speed rather than the exact rate or speed of the variations. In this paper, we propose to classfy propagation channel conditions into three categories as per the estimated channel variation rate: slow, medium, and high speed. These three categories reflect the trend of channel variation. Slow varying channel means that noticeable changes in their characteristics are observed after relatively long time is elapsed. High speed categoty means that significant changes in their channel properties are observed in very short time. That is, the acutal rate of channel variations is not important. The more relevent information to reflect the changes in their characteristics is how much they have been chaged actually. In this paper, we propose to use such relevent information based on CSI's intead of estimating the rate of channel variations directly. In other words, the trend of channel variations are divided to three groups of microscopic, mediumscopic, and macroscopic channel changes.

The proposed multi-stage link adaptation strategy is very simple. First, three stages of link adaptations are set up: microscopic, medium scopic, and macroscopic channel changes. For each stage, there exists the link adaptation algorithm optimized for the characteristic of each stage. For example, the link adaptation algorithm of the first stage for microscopic change in channel should be simple enough to be run fast. A simple up/down power control would be a good candidate for the link adaptation algorithm of this stage. In Stage 3, the optimal link adaptation algorithm of allocatioins in Section 3 can be used because macroscopic changes in channel are infrequent usually. Therefore, periodic runnings of the optimal allocation algorithm should be sufficient for Stage 3. Because the required duty cycle is pretty long in this case, there is no problem to run the optimal algorithm in real time. On the other hand, Stage 2 must compensate 
for the possible performance loss when the noticeable channel changes happen in a time shorter than Stage 3 running period. For this, the stage 2 kicks in and run the link adaptation algorithm optimized for this stage asynchronously if the accumulated channel variation from CSI's is larger than a threshold. Stage 1 keeps running during the call. On the other hand, the running period of Stage 3 is determined by the threshold $T h$. Fig. 2 shows the only simplified version of the flow chart of the proposed link adaptation scheme due to the space limitation.

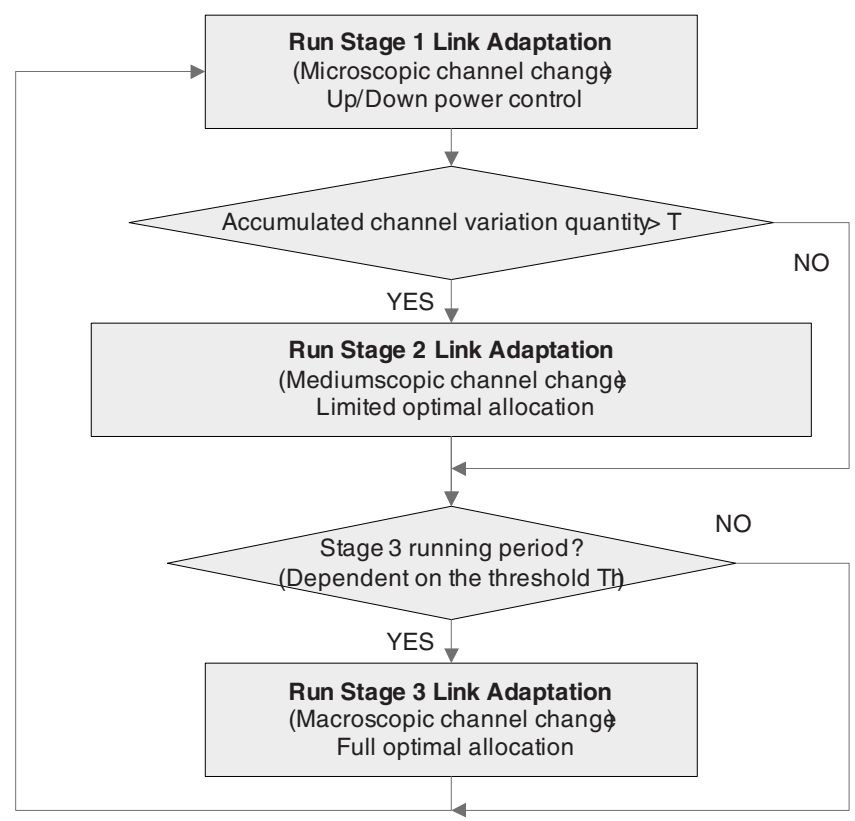

Fig. 2. The simplified flow chart of the proposed multi-stage link adaptation

\section{$5 \quad$ Simulation Results}

To show the effectiveness of the proposed multi-stage link adaptation scheme, the performance and complexity of the proposed method is compared with the optimal and sub-optimal ones in 6] and [8. Here we consider an OFDM system with several multi-stage switching rates and thresholds. Several mobile speeds of $0 \mathrm{~km} / \mathrm{h}, 3 \mathrm{~km} / \mathrm{h}, 30 \mathrm{~km} / \mathrm{h}, 60 \mathrm{~km} / \mathrm{h}, 90 \mathrm{~km} / \mathrm{h}$, and $120 \mathrm{~km} / \mathrm{h}$ are considered to simulate the various rates of channel variations. The basic unit of performing the link adaptation is multiple of frames.

The performance of the proposed scheme is shown in Fig. 3 together with the optimal one that is performing the link adaptation every frame. In practice, the optimal scheme is hardly finished in a frame, so that it cannot be used in the real time application. In addition, sub-optimal scheme of running the link adaptation over two frames is also shown in the same plot for the comparison purpose. Four 
different link adaptation duty cyle threshold values of 1, 0.1, 0.01, and 0.001 are simulated to show the effectiveness of the proposed multi-stage link adaptation scheme. As the threshold value increases, the duty cycle of Stage 3 is increased so that the total number of full link adapation operations is reduced. It results in much less complexity and sufficient cycle time for the real time operation. Note that Stage 1 of simple power control for microscopic channel variations and Stage 2 link adaptation for mediumscopic channel variations are running asynchronously regardless of the threshold values for Stage 3 duty cycle. Table 1 summarizes the total number of the full link adaptation operations to compare the complexity of the proposed scheme with the optimal one.

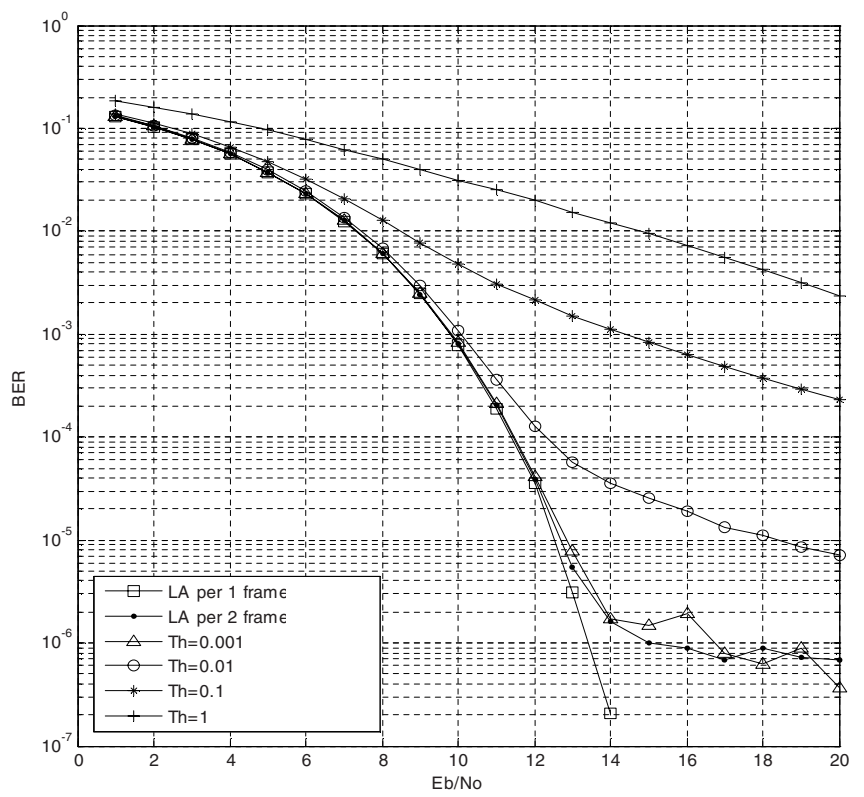

Fig. 3. The performance of the proposed method

Fig. 3 shows that the performance of the proposed multi-stage link adaptation scheme is close to the performance of the optimal method while considerable reduction in the total number of full link adaption operations is achieved as shown in Table 1

It is obvious that the proposed multi-stage link adaptation scheme can provide the real time operations in practical wirelss communication systems even without noticeable performance degradation. The performance gap between the proposed method and the optimal one will becomes noticeable eventually when the rate of channel variation is very high. In this case, however, correct CSI's cannot be available at BS side at the right timing because of CSI feedback loop delay. Even optimal method also suffers from it. 
Table 1. Simulation Parameters

\begin{tabular}{c|c|c}
\hline \multirow{2}{*}{ Speed } & \multicolumn{2}{|c}{ Duty cycle in frames } \\
\cline { 2 - 3 } & $T h=0.01$ & $T h=0.001$ \\
\hline $3 \mathrm{~km} / \mathrm{h}$ & 132 & 42 \\
$30 \mathrm{~km} / \mathrm{h}$ & 22 & 7 \\
$60 \mathrm{~km} / \mathrm{h}$ & 14 & 5 \\
$90 \mathrm{~km} / \mathrm{h}$ & 10 & 3 \\
$120 \mathrm{~km} / \mathrm{h}$ & 8 & 2 \\
\hline
\end{tabular}

\section{Conclusion}

In this paper, we considered the real time link adaptation strategy for downlink OFDM transmission. To satisfy the time variation of fading channel, a realtime heuristic multi-stage link adaptation strategy based on the determined channel variation speed using available CSI's was proposed. Switchig between optimized link adaptation stages were controlled adaptively. Simulation results show that the proposed multi-stage link adaptation scheme provides a solution that is considerably simpler to implement than the optimal method, and that is appropriate for the real time applications, while its performance is very close to the optimal solution.

\section{References}

1. Rohling, H., Grunheid, R.: Performance of an OFDM-TDMA Mobile communication system. Proc. IEEE Vehicular Technology Conference (1996) 1589-1593

2. Czylwik, R.: Adaptive OFDM for wideband radio channels. Proc. IEEE Globecom conference (1996) 713-718

3. Rhee, W., Cioffi, J.: Increase in capacity of multiuser OFDM system using dynamic subchannel allocation. Proc. IEEE Vehicular Technology Conference (2000) 1058-1089

4. Yin, H., Liu, H.: An Efficient Multiuser Loading Algorithm for OFDM-based Broadband Wireless Systems. Proc. IEEE Globecom conference (2000) 103-107

5. Chen, Y., Chen, J., Li P.: A fast suboptimal subcarrier, bit, and power allocation algorithm for multiuser OFDM-based systems. Proc. IEEE International Conference on Communications (2004) 3212-3216

6. Wong, C., Cheng, R., Letaief, K., Murch, R.: Mutliuser Sub-carrier Allocation for OFDM Transmission using Adaptive Modulation. Proc. IEEE Vehicular Technology Conference (1999) 479-483

7. Khun, H.: The Hungarian Method for the Assignment Problem. Naval Research Logistics Quarterly 2 (1955) 83-97

8. Wong, C., Tsui, C., Cheng, R., Letaief, K.: A real-time sub-carrier allocation scheme for multiple access downlink OFDM transmission. Proc. IEEE Vehicular Technology Conference (1999) 1124-1128 